\title{
POLYNOMIAL APPROXIMATION WITH POLLACZECK-LAGUERRE WEIGHTS ON THE REAL SEMIAXIS. A SURVEY*
}

\author{
GIUSEPPE MASTROIANNI ${ }^{\dagger}$, GRADIMIR V. MILOVANOVIĆ ${ }^{\ddagger}$, AND INCORONATA NOTARANGELO $§$
}

\section{Dedicated to Walter Gautschi on the occasion of his 90th birthday}

\begin{abstract}
This paper summarizes recent results on weighted polynomial approximations for functions defined on the real semiaxis. The function may grow exponentially both at 0 and at $+\infty$. We discuss orthogonal polynomials, polynomial inequalities, function spaces with new moduli of smoothness, estimates for the best approximation, Gaussian rules, and Lagrange interpolation with respect to the weight $w(x)=x^{\gamma} \mathrm{e}^{-x^{-\alpha}-x^{\beta}}(\alpha>0, \beta>1$, $\gamma \geq 0$ ).
\end{abstract}

Key words. orthogonal polynomials, weighted polynomial approximation, polynomial inequalities, Gaussian quadrature rules, Lagrange interpolation, Pollaczeck-Laguerre exponential weights

AMS subject classifications. 41A05, 41A10, 41A17, 41A25, 65D05, 65D32

1. Introduction. This paper is a short survey on weighted polynomial approximations of functions defined on the real semiaxis. The function may grow exponentially both at 0 and at $+\infty$. As far as we know, this topic has received attention in the literature only recently (see $[12,13,14,15,16])$. We consider weight functions of the form

$$
w(x)=x^{\gamma} \mathrm{e}^{-x^{-\alpha}-x^{\beta}}, \quad \alpha>0, \beta>1, \gamma \geq 0, \quad x \in(0,+\infty) .
$$

Even though $w$ can be seen as a combination of a Pollaczeck-type weight $\mathrm{e}^{-x^{-\alpha}}$ and a Laguerre-type weight $x^{\gamma} \mathrm{e}^{-x^{\beta}}$, one cannot investigate the problem by reducing it to a combination of a Pollaczeck-type case (on, say, $[0,1])$ and a Laguerre-type case $($ on $[1,+\infty)$ ).

We are going to present the main results concerning orthogonal polynomials, polynomial inequalities, function spaces with new moduli of smoothness, and estimates for the best polynomial approximation with respect to the weight $w$. We also pay due attention to Gaussian rules and Lagrange interpolation in weighted $L^{2}$-norms. The behaviour of the related Fourier sums and their discrete versions, the Lagrange polynomials, in the $L^{p}$-norms remains an open problem.

In the sequel $c, \mathcal{C}$ will stand for positive constants that may assume different values in each formula, and we shall write $\mathcal{C} \neq \mathcal{C}(a, b, \ldots)$ when $\mathcal{C}$ is independent of $a, b, \ldots$ Furthermore, $A \sim B$ means that if $A$ and $B$ are positive quantities depending on some parameters, then there exists a positive constant $\mathcal{C}$ independent of these parameters such that $(A / B)^{ \pm 1} \leq \mathcal{C}$. Finally, we denote by $\mathbb{P}_{m}$ the set of all algebraic polynomials of degree at most $m$. As usual $\mathbb{N}$, $\mathbb{Z}, \mathbb{R}$, will stand for the sets of all natural, integer, and real numbers, while $\mathbb{Z}^{+}$and $\mathbb{R}^{+}$denote the sets of positive integer and positive real numbers, respectively.

* Received May 7, 2018. Accepted August 20, 2018. Published online on November 15, 2018. Recommended by L. Reichel. The first author was supported in part by the University of Basilicata (local funds), the second author by the Serbian Academy of Sciences and Arts (No. \$-96), and the third author by the University of Basilicata (local funds) and by INdAM-GNCS.

$\dagger$ Department of Mathematics, Computer Sciences and Economics, University of Basilicata, Via dell'Ateneo Lucano 10, 85100 Potenza, Italy (giuseppe.mastroianni@unibas.it).

${ }_{\ddagger}^{\ddagger}$ Serbian Academy of Sciences and Arts, Belgrade, Serbia \& University of Niš, Faculty of Sciences and Mathematics, 18000 Niš, Serbia (gvm@mi.sanu.ac.rs).

${ }^{\S}$ Department of Mathematics, Computer Sciences and Economics, University of Basilicata, Via dell'Ateneo Lucano 10, 85100 Potenza, Italy (incoronata. notarangelo@unibas.it). 
2. Orthogonal polynomials. First of all we note that the weight $w$ defined by (1.1) can be reduced to a weight belonging to the class $\mathcal{F}\left(C^{2}+\right)$, introduced by Levin and Lubinsky in [7, pp. 7-8], by a linear transformation. Let us recall the definition of this class for the reader's convenience.

Let $I=(c, d)$ be an interval with $-\infty \leq c<0<d \leq+\infty$, and $\varrho: I \in \mathbb{R}$ be a weight function with $\varrho=\mathrm{e}^{-Q}, Q: I \in[0,+\infty)$ satisfying the following properties:

(i) $Q^{\prime}$ is continuous in $I$ and $Q(0)=0$;

(ii) $Q^{\prime \prime}$ exists and is positive in $I \backslash\{0\}$;

(iii) $\lim _{x \rightarrow c^{+}} Q(x)=\lim _{x \rightarrow d^{-}} Q(x)=\infty$;

(iv) the function

$$
T(x)=\frac{x Q^{\prime}(x)}{Q(x)}, \quad x \in I \backslash\{0\},
$$

is quasi-decreasing in $(c, 0)$ and quasi-increasing in $(0, d)$ with

$$
T(x) \geq \Lambda>1, \quad x \in I \backslash\{0\} ;
$$

(v) there exist $C_{1}, C_{2}>0$ and a compact subinterval $J \subseteq I$ such that

$$
\frac{Q^{\prime \prime}(x)}{\left|Q^{\prime}(x)\right|} \leq C_{1} \frac{\left|Q^{\prime}(x)\right|}{Q(x)}, \quad \text { a.e. } \quad x \in I \backslash\{0\},
$$

and

$$
\frac{Q^{\prime \prime}(x)}{\left|Q^{\prime}(x)\right|} \geq C_{2} \frac{\left|Q^{\prime}(x)\right|}{Q(x)}, \quad \text { a.e. } \quad x \in I \backslash J
$$

Then we say that $\varrho \in \mathcal{F}\left(C^{2}+\right)$.

With the previous notation, we can state the following lemma.

LEMMA 2.1 (see [16, pp. 817-818]). Letting $w$ be the weight in (1.1), there exists a $\lambda>0$ such that the weight $\widetilde{w}$ defined as

$$
\widetilde{w}(y)=\mathrm{e}^{-Q(y)}, \quad y \in(-\lambda,+\infty),
$$

with

$$
Q(y)=\frac{1}{(y+\lambda)^{\alpha}}+(y+\lambda)^{\beta}-\gamma \log (y+\lambda)-\lambda^{-\alpha}-\lambda^{\beta}+\gamma \log (\lambda),
$$

belongs to the class $\mathcal{F}\left(C^{2}+\right)$.

Therefore, we have that $w(y)=\mathcal{C} \widetilde{w}(y+\lambda)$, where $\lambda$ is the unique positive zero of

$$
q^{\prime}(x)=-\alpha x^{-\alpha-1}+\beta x^{\beta-1}-\gamma x^{-1} .
$$

We can deduce the properties of the orthogonal polynomials with respect to our weight $w$ from the results obtained by Levin and Lubinsky using the inverse transformation. The Mhaskar-Rakhmanov-Saff (MRS) numbers, $\varepsilon_{\tau}=\varepsilon_{\tau}(w)$ and $a_{\tau}=a_{\tau}(w)$, related to

$$
w(x)=\mathrm{e}^{-q(x)}, \quad q(x)=x^{-\alpha}+x^{\beta}-\gamma \log (x),
$$

are defined by

$$
\tau=\frac{1}{\pi} \int_{\varepsilon_{\tau}}^{a_{\tau}} \frac{x q^{\prime}(x)}{\sqrt{\left(a_{\tau}-x\right)\left(x-\varepsilon_{\tau}\right)}} \mathrm{d} x
$$


and

$$
0=\frac{1}{\pi} \int_{\varepsilon_{\tau}}^{a_{\tau}} \frac{q^{\prime}(x)}{\sqrt{\left(a_{\tau}-x\right)\left(x-\varepsilon_{\tau}\right)}} \mathrm{d} x .
$$

Proposition 2.2 (see [16, pp. 820] and [7, p. 13]). For $\tau>0$, $\varepsilon_{\tau}$ is a decreasing function, and $a_{\tau}$ is an increasing function of $\tau$, and

$$
\lim _{\tau \rightarrow+\infty} \varepsilon_{\tau}=0, \quad \lim _{\tau \rightarrow+\infty} a_{\tau}=+\infty,
$$

with

$$
\varepsilon_{\tau} \sim\left(\frac{\sqrt{a_{\tau}}}{\tau}\right)^{\frac{1}{\alpha+1 / 2}}
$$

and

$$
a_{\tau} \sim \tau^{1 / \beta}
$$

Let us denote by $\left\{p_{m}(w)\right\}_{m \in \mathbb{N}}$ the sequence of orthonormal polynomials defined by

$$
p_{m}(w, x)=\gamma_{m} x^{m}+\text { lower degree terms, } \quad \gamma_{m}=\gamma_{m}(w)>0,
$$

and

$$
\int_{0}^{+\infty} p_{m}(w, x) p_{n}(w, x) w(x) \mathrm{d} x=\delta_{m, n} .
$$

The zeros of $p_{m}(w)$ lie in the MRS interval associated with $\sqrt{w}$. Here and for the rest of the paper, we use the notation $\varepsilon_{\tau}=\varepsilon_{\tau}(\sqrt{w})$ and $a_{\tau}=a_{\tau}(\sqrt{w})$, taking into account that, by definition, $\varepsilon_{\tau}(\sqrt{w})=\varepsilon_{2 \tau}(w)$ and $a_{\tau}(\sqrt{w})=a_{2 \tau}(w)$. The next proposition provides further information concerning the distribution of these zeros.

PROPOSITION 2.3 (see [14, pp. 1656-1657] and [7, pp. 312-324]). The zeros of $p_{m}(w)$ are located as

$$
\varepsilon_{m}<x_{1}<x_{2}<\cdots<x_{m}<a_{m}
$$

with

$$
x_{1}-\varepsilon_{m} \sim \delta_{m}, \quad \delta_{m} \sim\left(\frac{\sqrt{a_{m}}}{m}\right)^{\frac{2}{3}\left(\frac{2 \alpha+3}{2 \alpha+1}\right)} \sim m^{-\frac{2}{3}\left(\frac{2 \alpha+3}{2 \alpha+1}\right)\left(1-\frac{1}{2 \beta}\right)}
$$

and

$$
a_{m}-x_{m} \sim a_{m} m^{-2 / 3} \sim m^{\frac{1}{\beta}-\frac{2}{3}}
$$

where the constants in " $\sim$ " are independent of $m$.

The distance between two consecutive zeros $\Delta x_{k}=x_{k+1}-x_{k}$ can be estimated by

$$
\Delta x_{k} \sim \Psi_{m}\left(x_{k}\right), \quad k=1, \ldots, m-1,
$$

where

$$
\Psi_{m}\left(x_{k}\right)=\frac{a_{m} x_{k}}{m \sqrt{\left(x_{k}-\varepsilon_{m}\right)\left(a_{m}-x_{k}\right)}}
$$

and the constants in “ $\sim$ " are independent of $k$ and $m$. 
Now, letting $\theta \in(0,1)$ be fixed, we define two indexes $j_{1}=j_{1}(m)$ and $j_{2}=j_{2}(m)$ as

$$
x_{j_{1}}=\max _{1 \leq k \leq m}\left\{x_{k}: x_{k} \leq \varepsilon_{\theta m}\right\} \quad \text { and } \quad x_{j_{2}}=\min _{1 \leq k \leq m}\left\{x_{k}: x_{k} \geq a_{\theta m}\right\} .
$$

For the sake of completeness, if $\left\{x_{k}: x_{k} \leq \varepsilon_{\theta m}\right\}$ or $\left\{x_{k}: x_{k} \geq a_{\theta m}\right\}$ are empty, then we set $x_{j_{1}}=x_{1}$ or $x_{j_{2}}=x_{m}$, respectively.

From Proposition 2.3, it follows that

$$
\Delta x_{k} \sim \frac{\sqrt{a_{m}}}{m} \sqrt{x_{k}}, \quad k=j_{1}, \ldots, j_{2} .
$$

Let

$$
\lambda_{m}(w, x)=\left(\sum_{k=0}^{m-1} p_{m}^{2}(w, x)\right)^{-1}
$$

be the $m$ th Christoffel function and

$$
\lambda_{k}(w)=\lambda_{m}\left(w, x_{k}\right), \quad k=1, \ldots, m,
$$

be the Christoffel numbers related to $w$.

Proposition 2.4 (see [7, p. 257]). We have

$$
\lambda_{m}(w, x) \sim \Psi_{m}(x) w(x), \quad x \in\left[\varepsilon_{m}, a_{m}\right],
$$

where $\Psi_{m}$ is given by

$$
\Psi_{m}(x)=\frac{a_{m} x}{m \sqrt{\left(x-\varepsilon_{m}+\delta_{m}\right)\left(a_{m}-x+a_{m} m^{-2 / 3}\right)}}
$$

and the constants in “ $\sim$ " are independent of $m$.

In particular, for $\theta \in(0,1)$, we get

$$
\lambda_{m}(w, x) \sim \frac{\sqrt{a_{m}}}{m} \sqrt{x} w(x), \quad x \in\left[\varepsilon_{\theta m}, a_{\theta m}\right] .
$$

From the numerical point of view, in order to compute the zeros of $p_{m}(w)$ and the Christoffel numbers, we use a procedure given in [14] (see also [18, §4.2]) and the MATHEMATICA package OrthogonalPolynomials (cf. [3] and [19]), which is freely downloadable from the website: http://www.mi.sanu.ac.rs/ gvm/.

For the sake of brevity we omit the description of the numerical procedures for the computation of the zeros of $p_{m}(w)$, the Christoffel numbers, and the Mhaskar-Rahmanov-Saff numbers $\varepsilon_{m}$ and $a_{m}$. The interested reader can find all the details about these procedures in [14, pp. 1676-1680] (cf. [15]).

The following estimates are crucial tools in order to study the convergence of several approximation processes.

PROPOSITION 2.5 (see [7, pp. 325 and 360]). We have

$$
\begin{gathered}
\sup _{x \in(0,+\infty)}\left|p_{m}(w, x)\right| \sqrt{w(x)} \sqrt[4]{\left|\left(a_{m}-x\right)\left(x-\varepsilon_{m}\right)\right|} \sim 1, \\
\sup _{x \in(0,+\infty)}\left|p_{m}(w, x)\right| \sqrt{w(x)} \sim m^{\frac{1}{6}\left(1-\frac{1}{2 \beta}\right)\left(\frac{2 \alpha+3}{2 \alpha+1}\right)},
\end{gathered}
$$


and

$$
\frac{1}{\left|p_{m}^{\prime}\left(w, x_{k}\right)\right| \sqrt{w\left(x_{k}\right)}} \sim \Delta x_{k} \sqrt[4]{\left(a_{m}-x\right)\left(x-\varepsilon_{m}\right)}
$$

where the constants in " $\sim$ " are independent of $m$.

PROPOSITION 2.6 (cf. [7, p. 25]). For the leading coefficient of $p_{m}(w)$, we have

$$
\gamma_{m}=\frac{1}{\sqrt{2 \pi}}\left(\frac{4}{a_{m}+\varepsilon_{m}}\right)^{m+\frac{1}{2}} \exp \left(\frac{1}{\pi} \int_{\varepsilon_{m}}^{a_{m}} \frac{q(x)}{\sqrt{\left(a_{m}-x\right)\left(x-\varepsilon_{m}\right)}} \mathrm{d} x\right)(1+o(1)),
$$

where $q(x)=\frac{1}{2}\left(x^{-\alpha}+x^{\beta}-\gamma \log x\right)$.

3. Polynomial inequalities. Letting $w$ be given by (1.1), $x \in \mathbb{R}_{+}$, we introduce the weight function

$$
u(x)=x^{\delta} \sqrt{w(x)}, \quad \delta \in \mathbb{R} .
$$

In the sequel, by a slight abuse of notation, we denote by $\|\cdot\|_{p}$ the quasinorm of the $L^{p}$-spaces for $0<p<1$ defined in the usual way.

Lemma 3.1 (see [16, p. 809]). Let $\delta \in \mathbb{R}$ and $n=m+\lceil|\delta|\rceil$. For any $P_{m} \in \mathbb{P}_{m}$ with $0<p \leq \infty$, we have

$$
\left\|P_{m} u\right\|_{p} \leq \mathcal{C}\left\|P_{m} u\right\|_{L^{p}\left[\varepsilon_{n}, a_{n}\right]},
$$

where $\mathcal{C} \neq \mathcal{C}\left(m, P_{m}\right)$ and $\varepsilon_{n}, a_{n}$ are defined by (2.1) and (2.2).

On the other hand, for any $s>1$, we have

$$
\left\|P_{m} u\right\|_{L^{p}\left(\mathbb{R}_{+} \backslash\left[\varepsilon_{s m}, a_{s m}\right]\right)} \leq \mathcal{C} \mathrm{e}^{-c m^{\nu}}\left\|P_{m} u\right\|_{p},
$$

where

$$
\nu=\left(1-\frac{1}{2 \beta}\right) \frac{2 \alpha}{2 \alpha+1}
$$

and $\mathcal{C}$ and $c$ are independent of $m$ and $P_{m}$.

For the rest of the paper, let

$$
\varphi(x)=\sqrt{x} .
$$

The following lemma is of independent interest and gives rise to a useful procedure for verifying polynomial inequalities.

LEMMA 3.2 (see [16, p. 809]). For a sufficiently large $m$ (say $m \geq m_{0}$ ), there exists a polynomial $R_{\ell m} \in \mathbb{P}_{\ell m}$, with $\ell$ a fixed integer, such that

$$
R_{\ell m}(x) \sim w(x)
$$

and

$$
\left|R_{\ell m}^{\prime}(x)\right| \varphi(x) \leq \mathcal{C} \frac{m}{\sqrt{a_{m}}} w(x)
$$

for $x \in\left[\varepsilon_{m}, a_{m}\right]$, where $\varepsilon_{m}=\varepsilon_{m}(w)$ and $a_{m}=a_{m}(w)$ are defined by (2.1) and (2.2). The constants in " $\sim$ " and $\mathcal{C}$ are independent of $m$. 
By Lemmas 3.1 and 3.2 we reduce the problem for the polynomial inequalities related to the weight $u$ on $(0,+\infty)$ to analogous inequalities on bounded intervals with Jacobi weights. In fact, we get:

THEOREM 3.3 (see $\left[16\right.$, p. 810]). Let $0<p \leq \infty$. Then, for any $P_{m} \in \mathbb{P}_{m}$, we have

$$
\left\|P_{m}^{\prime} \varphi u\right\|_{p} \leq \mathcal{C} \frac{m}{\sqrt{a_{m}}}\left\|P_{m} u\right\|_{p}
$$

and

$$
\left\|P_{m}^{\prime} u\right\|_{p} \leq \mathcal{C} \frac{m}{\sqrt{\varepsilon_{m} a_{m}}}\left\|P_{m} u\right\|_{p}
$$

where $\mathcal{C} \neq \mathcal{C}\left(m, P_{m}\right)$.

We want to emphasize that the presence of the algebraic factor $x^{\delta}$ in the definition of $u$ allows us to iterate the Bernstein inequality (3.3) as follows:

$$
\left\|P_{m}^{(r)} \varphi^{r} u\right\|_{p} \leq \mathcal{C}\left(\frac{m}{\sqrt{a_{m}}}\right)^{r}\left\|P_{m} u\right\|_{p}
$$

for $1 \leq r \in \mathbb{Z}$. Also, the factor

$$
\frac{m}{\sqrt{\varepsilon_{m} a_{m}}} \sim\left(\frac{m}{\sqrt{a_{m}}}\right)^{\frac{2 \alpha+2}{2 \alpha+1}}=\left(\frac{m}{\sqrt{a_{m}}}\right)^{1+\frac{1}{2 \alpha+1}}
$$

in the Markoff inequality (3.4) is smaller than the one appearing in the analogous inequality (see [17])

$$
\left\|P_{m}^{\prime} w_{\beta}\right\|_{p} \leq \mathcal{C}\left(\frac{m}{\sqrt{a_{m}}}\right)^{2}\left\|P_{m} w_{\beta}\right\|_{p}
$$

with the generalized Laguerre weight $w_{\beta}(x)=\mathrm{e}^{-x^{\beta}}$ on $(0,+\infty)$, whereas the factors of the Bernstein inequalities for the weights $u$ and $w_{\beta}$ are the same.

Using standard arguments, the Markoff inequality (3.4) can be deduced from the Bernstein inequality (3.3) and the Schur inequality stated in the following theorem.

THEOREM 3.4 (see $\left[16\right.$, p. 810]). Let $0<p \leq \infty$. Then, for any $P_{m} \in \mathbb{P}_{m}$, we have

$$
\left\|P_{m} u\right\|_{p} \leq \mathcal{C}\left(\frac{m}{\sqrt{a_{m}}}\right)^{\frac{\delta}{\alpha+1 / 2}}\left\|P_{m} v_{\delta} u\right\|_{p},
$$

where $v_{\delta}(x)=x^{\delta}$ and $\mathcal{C} \neq \mathcal{C}\left(m, P_{m}\right)$.

In analogy with the Bernstein and Markoff inequalities, we give two versions of the Nikolskii inequality.

THEOREM 3.5 (see [16, p. 810]). Let $0<p<q \leq \infty$. Then, for any $P_{m} \in \mathbb{P}_{m}$, we have

$$
\left\|P_{m} \varphi^{\frac{1}{p}-\frac{1}{q}} u\right\|_{q} \leq \mathcal{C}\left(\frac{m}{\sqrt{a_{m}}}\right)^{\frac{1}{p}-\frac{1}{q}}\left\|P_{m} u\right\|_{p}
$$

and

$$
\left\|P_{m} u\right\|_{q} \leq \mathcal{C}\left(\frac{m}{\sqrt{\varepsilon_{m} a_{m}}}\right)^{\frac{1}{p}-\frac{1}{q}}\left\|P_{m} u\right\|_{p}
$$

where $\mathcal{C} \neq \mathcal{C}\left(m, P_{m}\right)$.

In analogy with different weighted polynomial inequalities, the factor $m / \sqrt{\varepsilon_{m} a_{m}}$ in the second Nikolskii inequality is the same as the one appearing in the Markoff inequality. 
4. Function spaces, $\boldsymbol{K}$-functionals, and moduli of smoothness. Let us now define some function spaces related to the weight $u$ (see [13, pp. 168-172]). By $L_{u}^{p}, 1 \leq p<\infty$, we denote the set of all measurable functions $f$ such that

$$
\|f\|_{L_{u}^{p}}:=\|f u\|_{p}=\left(\int_{0}^{+\infty}|f u|^{p}(x) \mathrm{d} x\right)^{1 / p}<\infty,
$$

while, for $p=\infty$, by a slight abuse of notation, we set

$$
L_{u}^{\infty}=C_{u}=\left\{f \in C^{0}(0,+\infty): \lim _{x \rightarrow 0^{+}} f(x) u(x)=0=\lim _{x \rightarrow+\infty} f(x) u(x)\right\}
$$

with the norm

$$
\|f\|_{L_{u}^{\infty}}:=\|f u\|_{\infty}=\sup _{x \in(0,+\infty)}|f(x) u(x)| .
$$

For smoother functions we introduce the Sobolev-type spaces

$$
W_{r}^{p}(u)=\left\{f \in L_{u}^{p}: f^{(r-1)} \in A C(0,+\infty),\left\|f^{(r)} \varphi^{r} u\right\|_{p}<\infty\right\},
$$

where $1 \leq p \leq \infty, 1 \leq r \in \mathbb{Z}^{+}, \varphi(x):=\sqrt{x}$, and $A C(0,+\infty)$ denotes the set of all absolutely continuous functions on $(0,+\infty)$. We equip these spaces with the norm

$$
\|f\|_{W_{r}^{p}(u)}=\|f u\|_{p}+\left\|f^{(r)} \varphi^{r} u\right\|_{p} .
$$

To characterize some subspaces of $L_{u}^{p}$, we introduce the following moduli of smoothness. Let us consider the intervals

$$
\mathcal{I}_{h}(c)=\left[h^{1 /(\alpha+1 / 2)}, \frac{c}{h^{1 /(\beta-1 / 2)}}\right],
$$

with $\alpha$ and $\beta$ in (3.1), $h>0$ sufficiently small, and $c>1$ an arbitrary but fixed constant. For any $f \in L_{u}^{p}, 1 \leq p \leq \infty, r \geq 1$, and $t>0$ sufficiently small (say $t<t_{0}$ ), we set

$$
\Omega_{\varphi}^{r}(f, t)_{u, p}=\sup _{0<h \leq t}\left\|\Delta_{h \varphi}^{r}(f) u\right\|_{L^{p}\left(\mathcal{I}_{h}(c)\right)},
$$

where

$$
\Delta_{h \varphi}^{r} f(x)=\sum_{i=0}^{r}(-1)^{i}\left(\begin{array}{l}
r \\
i
\end{array}\right) f(x+(r-i) h \varphi(x)), \quad \varphi(x)=\sqrt{x} .
$$

Moreover, we introduce the following $K$-functional

$$
K\left(f, t^{r}\right)_{u, p}=\inf _{g \in W_{r}^{p}(u)}\left\{\|(f-g) u\|_{p}+t^{r}\left\|g^{(r)} \varphi^{r} u\right\|_{p}\right\}
$$

and its main part

$$
\widetilde{K}\left(f, t^{r}\right)_{u, p}=\sup _{0<h \leq t} \inf _{g \in W_{r}^{p}(u)}\left\{\|(f-g) u\|_{L^{p}\left(\mathcal{I}_{h}(c)\right)}+h^{r}\left\|g^{(r)} \varphi^{r} u\right\|_{L^{p}\left(\mathcal{I}_{h}(c)\right)}\right\} .
$$

The main part of the $K$-functional is equivalent to the main part of the previous modulus of smoothness as the following lemma shows. 
Lemma 4.1 (see [13, p. 171]). Let $r \geq 1$ and $0<t<t_{0}$ for some $t_{0}<1$. Then, for any $f \in L_{u}^{p}, 1 \leq p \leq \infty$, we have

$$
\Omega_{\varphi}^{r}(f, t)_{u, p} \sim \widetilde{K}\left(f, t^{r}\right)_{u, p},
$$

where the constants in " $\sim$ " are independent of $f$ and $t$.

Then we define the complete $r$ th modulus of smoothness by

$$
\begin{aligned}
\omega_{\varphi}^{r}(f, t)_{u, p}=\Omega_{\varphi}^{r}(f, t)_{u, p} & +\inf _{q \in \mathbb{P}_{r-1}}\|(f-q) u\|_{L^{p}\left(0, t^{1 /(\alpha+1 / 2)}\right]} \\
& +\inf _{q \in \mathbb{P}_{r-1}}\|(f-q) u\|_{L^{p}\left[c t^{-1 /(\beta-1 / 2)},+\infty\right)},
\end{aligned}
$$

with $c>1$ a fixed constant. We emphasize that the behaviour of $\omega_{\varphi}^{r}(f, t)_{u, p}$ is independent of the constant $c$. Moreover, the following lemma shows that this modulus of smoothness is equivalent to the $K$-functional.

Lemma 4.2 (see [13, p. 172]). Let $r \geq 1$ and $0<t<t_{0}$ for some $t_{0}<1$. Then, for any $f \in L_{u}^{p}, 1 \leq p \leq \infty$, we have

$$
\omega_{\varphi}^{r}(f, t)_{u, p} \sim K\left(f, t^{r}\right)_{u, p}
$$

where the constants in " $\sim$ " are independent of $f$ and $t$.

By means of the main part of the modulus of smoothness, for $1 \leq p \leq \infty$, we can define the Zygmund-type spaces

$$
Z_{s}^{p}(u)=\left\{f \in L_{u}^{p}: \sup _{t>0} \frac{\Omega_{\varphi}^{r}(f, t)_{u, p}}{t^{s}}<\infty, r>s\right\}
$$

$s \in \mathbb{R}^{+}$, with the norm

$$
\|f\|_{Z_{s}^{p}(u)}=\|f\|_{L_{u}^{p}}+\sup _{t>0} \frac{\Omega_{\varphi}^{r}(f, t)_{u, p}}{t^{s}} .
$$

We remark that, in the definition of $Z_{s}^{p}(u)$, the main part of the $r$ th modulus of smoothness $\Omega_{\varphi}^{r}(f, t)_{u, p}$ can be replaced by the complete modulus $\omega_{\varphi}^{r}(f, t)_{u, p}$ as can be deduced from Theorem 5.1 in next section.

\section{Weighted approximation and embedding theorems.}

5.1. Estimates for the best weighted approximation. Let us denote by

$$
E_{m}(f)_{u, p}=\inf _{P \in \mathbb{P}_{m}}\|(f-P) u\|_{p}
$$

the error of the best polynomial approximation of a function $f \in L_{u}^{p}, 1 \leq p \leq \infty$. The following Jackson, weak Jackson, and Stechkin inequalities hold true.

THEOREM 5.1 (see [13, p. 173]). For any $f \in L_{u}^{p}, 1 \leq p \leq \infty$, and $m>r \geq 1$, we have

$$
E_{m}(f)_{u, p} \leq \mathcal{C} \omega_{\varphi}^{r}\left(f, \frac{\sqrt{a_{m}}}{m}\right)_{u, p}
$$

and, assuming $\Omega_{\varphi}^{r}(f, t)_{u, p} t^{-1} \in L^{1}[0,1]$,

$$
E_{m}(f)_{u, p} \leq \mathcal{C} \int_{0}^{\frac{\sqrt{a_{m}}}{m}} \frac{\Omega_{\varphi}^{r}(f, t)_{u, p}}{t} \mathrm{~d} t, \quad r<m .
$$


Finally, for any $f \in L_{u}^{p}, 1 \leq p \leq \infty$, we get

$$
\omega_{\varphi}^{r}\left(f, \frac{\sqrt{a_{m}}}{m}\right)_{u, p} \leq \mathcal{C}\left(\frac{\sqrt{a_{m}}}{m}\right)^{r} \sum_{i=0}^{m}\left(\frac{i}{\sqrt{a_{i}}}\right)^{r} \frac{E_{i}(f)_{u, p}}{i} .
$$

In any case $\mathcal{C}$ is independent of $m$ and $f$.

In particular, for any $f \in W_{r}^{p}(u), 1 \leq p \leq+\infty$, we obtain

$$
E_{m}(f)_{u, p} \leq \mathcal{C}\left(\frac{\sqrt{a_{m}}}{m}\right)^{r}\left\|f^{(r)} \varphi^{r} u\right\|_{p}, \quad \mathcal{C} \neq \mathcal{C}(m, f)
$$

whereas, for any $f \in Z_{s}^{p}(u), 1 \leq p \leq+\infty$, we get

$$
E_{m}(f)_{u, p} \leq \mathcal{C}\left(\frac{\sqrt{a_{m}}}{m}\right)^{s} \sup _{t>0} \frac{\Omega_{\varphi}^{r}(f, t)_{u, p}}{t^{s}}, \quad r>s, \quad \mathcal{C} \neq \mathcal{C}(m, f) .
$$

From (5.1), (5.2), and (5.4) we deduce the following equivalences

$$
\lim _{m} \omega_{\varphi}\left(f, \frac{\sqrt{a_{m}}}{m}\right)_{u, p}=0 \quad \Leftrightarrow \quad \lim _{m} E_{m}(f)_{u, p}=0
$$

and

$$
\|f u\|_{p}+\sup _{t>0} \frac{\Omega_{\varphi}^{r}(f, t)_{u, p}}{t^{s}} \sim\|f u\|_{p}+\sup _{m \geq 1}\left(\frac{m}{\sqrt{a_{m}}}\right)^{s} E_{m}(f)_{u, p}
$$

for $1 \leq p \leq \infty$ and $r>s$.

5.2. Embedding theorems. Now, using Theorem 5.1, the dyadic decomposition, the Nikolskii inequalities (3.5) and (3.6), we can show some embedding theorems, connecting different subspaces of $L_{u}^{p}$.

THEOREM 5.2 (see [12, p. 159]). For any $f \in L_{u}^{p}, 1 \leq p<\infty$, such that

$$
\int_{0}^{1} \frac{\Omega_{\varphi}^{r}(f, t)_{u, p}}{t^{1+\eta / p}} \mathrm{~d} t<\infty
$$

where $\eta=(2 \alpha+2) /(2 \alpha+1)$, we have

$$
\begin{aligned}
& E_{m}(f)_{u, \infty} \leq \mathcal{C} \int_{0}^{\frac{\sqrt{a_{m}}}{m}} \frac{\Omega_{\varphi}^{r}(f, t)_{u, p}}{t^{1+\eta / p}} \mathrm{~d} t, \\
& \Omega_{\varphi}^{r}\left(f, \frac{\sqrt{a_{m}}}{m}\right)_{u, \infty} \leq \mathcal{C} \int_{0}^{\frac{\sqrt{a_{m}}}{m}} \frac{\Omega_{\varphi}^{r}(f, t)_{u, p}}{t^{1+\eta / p}} \mathrm{~d} t, \\
&\|f u\|_{\infty} \leq \mathcal{C}\left\{\|f u\|_{p}+\int_{0}^{1} \frac{\Omega_{\varphi}^{r}(f, t)_{u, p}}{t^{1+\eta / p}} \mathrm{~d} t\right\},
\end{aligned}
$$

where $\mathcal{C}$ depends only on $r$. 
In the following theorem, we replace $\eta / p$ by $1 / p$.

THEOREM 5.3 (see [12, pp. 159-160]). For any $f \in L_{u}^{p}, 1 \leq p<\infty$, such that

$$
\int_{0}^{1} \frac{\Omega_{\varphi}^{r}(f, t)_{u, p}}{t^{1+1 / p}} \mathrm{~d} t<\infty
$$

we have

$$
\begin{aligned}
& E_{m}(f)_{\varphi^{1 / p} u, \infty} \leq \mathcal{C} \int_{0}^{\frac{\sqrt{a_{m}}}{m}} \frac{\Omega_{\varphi}^{r}(f, t)_{u, p}}{t^{1+1 / p}} \mathrm{~d} t, \\
& \Omega_{\varphi}^{r}\left(f, \frac{\sqrt{a_{m}}}{m}\right)_{\varphi^{1 / p} u, \infty} \leq \mathcal{C} \int_{0}^{\frac{\sqrt{a_{m}}}{m}} \frac{\Omega_{\varphi}^{r}(f, t)_{u, p}}{t^{1+1 / p} \mathrm{~d} t,} \\
&\left\|f \varphi^{1 / p} u\right\|_{\infty} \leq \mathcal{C}\left\{\|f u\|_{p}+\int_{0}^{1} \frac{\Omega_{\varphi}^{r}(f, t)_{u, p}}{t^{1+1 / p}} \mathrm{~d} t\right\},
\end{aligned}
$$

where $\mathcal{C}$ depends only on $r$.

From Theorem 5.3 we can easily deduce the following corollary, which is useful in several contexts.

COROLlary 5.4 (see [12, p. 160]). If $f \in L_{u}^{p}, 1 \leq p<\infty$, is such that

$$
\int_{0}^{1} \frac{\Omega_{\varphi}^{r}(f, t)_{u, p}}{t^{1+1 / p}} \mathrm{~d} t<\infty
$$

then $f$ is continuous on $(0,+\infty)$.

6. Quadrature rules and Lagrange interpolation. Here we are going to show a slight extension of the results proved in [14] for $\gamma=0$.

6.1. Gaussian formulas. The Gaussian rule related to the weight $w(x)=x^{\gamma} \mathrm{e}^{-x^{-\alpha}-x^{\beta}}$ can be defined by the equality

$$
\int_{0}^{+\infty} P_{2 m-1}(x) w(x) \mathrm{d} x=\sum_{k=1}^{m} \lambda_{k}(w) P_{2 m-1}\left(x_{k}\right),
$$

where $x_{k}$ are the zeros of $p_{m}(w), \lambda_{k}(w)$ are the Christoffel numbers, and (6.1) has to hold for any polynomial $P_{2 m-1} \in \mathbb{P}_{2 m-1}$. Thus the error of the Gaussian rule for any continuous function $f$ is given by

$$
e_{m}(f)=\int_{0}^{+\infty} f(x) w(x) \mathrm{d} x-\sum_{k=1}^{m} \lambda_{k}(w) f\left(x_{k}\right) .
$$

Let us consider the weight

$$
\sigma(x)=(1+x)^{\delta} w^{a}(x), \quad \delta \geq 0,0<a \leq 1 .
$$

Naturally, taking also into account Lemma 3.1, the results of Sections 3 and 4 hold with $u$ replaced by $\sigma$. If we assume that $f \in C_{\sigma}$, then we can write

$$
\left|\sum_{k=1}^{m} \lambda_{k}(w) f\left(x_{k}\right)\right| \leq\|f \sigma\|_{\infty} \sum_{k=1}^{m} \frac{\lambda_{k}(w)}{\sigma\left(x_{k}\right)} \leq \mathcal{C}\|f \sigma\|_{\infty} \int_{0}^{+\infty} \frac{w(x)}{\sigma(x)} \mathrm{d} x
$$


and the next proposition easily follows.

Proposition 6.1 (cf. [14, p. 1660]). If $w / \sigma \in L^{1}$, then, for any $f \in C_{\sigma}$, we have

$$
\left|e_{m}(f)\right| \leq \mathcal{C} E_{2 m-1}(f)_{\sigma, \infty},
$$

where $\mathcal{C} \neq \mathcal{C}(m, f)$.

This proposition generalizes a result due to Uspensky [20], who first proved convergence of Gaussian rules on unbounded intervals related to Laguerre and Hermite weights (see also [9, pp. 341-345] and [11]).

Notice that the assumption $w / \sigma \in L^{1}$ in Proposition 6.1 is fulfilled if $a=1$ and $\delta>1$ or if $a<1$ and $\delta$ is arbitrary. The error estimate (6.3) implies convergence of the Gaussian rules for any $f \in C_{\sigma}$. For a smoother function, for instance $f \in W_{r}^{\infty}(\sigma)$, by (6.3) and (5.3), we obtain

$$
\left|e_{m}(f)\right| \leq \mathcal{C}\left(\frac{\sqrt{a_{m}}}{m}\right)^{r}\left\|f^{(r)} \varphi^{r} \sigma\right\|_{\infty}
$$

where $\mathcal{C} \neq \mathcal{C}(m, f)$ and $a_{m} \sim m^{1 / \beta}$.

Thus, a natural question is how to establish the degree of convergence of $e_{m}(f)$ if the function $f$ is infinitely differentiable, i.e., $f \in C^{\infty}\left(\mathbb{R}_{+}\right)$. We recall that Aljarrah [1,2] showed estimates of $e_{m}(f)$ related to Hermite or Freud weights for analytic functions in some domains of the complex plane containing the quadrature nodes. For precise estimates, considering the same class of functions and different weights, we refer to [8]. Here we consider the case of infinitely differentiable functions on $\mathbb{R}_{+}$with the condition that $\left(f^{(m)} \sigma\right)(x)$ is uniformly bounded with respect to $m$ and $x$. We note that the derivatives of the function can increase exponentially for $x \rightarrow 0$ and $x \rightarrow+\infty$.

THEOREM 6.2 (cf. [14, p. 1660]). Let $\sigma$ be the weight in (6.2) with $0<a<1$ and $\delta$ arbitrary. For any infinitely differentiable function $f$, if $K(f):=\sup _{m}\left\|f^{(m)} \sigma\right\|_{\infty}<+\infty$, then we have

$$
\left|e_{m}(f)\right| \leq \mathcal{C} K(f) \Gamma_{m}, \quad \lim _{m} \sqrt[2 m]{\Gamma_{m}}=0 .
$$

In order to study the behaviour of the Gaussian rule in the Sobolev spaces $W_{r}^{1}(w)$, it is natural to investigate whether estimates of the form

$$
\left|e_{m}(f)\right| \leq \mathcal{C} \frac{\sqrt{a_{m}}}{m}\left\|f^{\prime} \varphi w\right\|_{1}, \quad \mathcal{C} \neq \mathcal{C}(m, f), \quad f \in W_{1}^{1}(w),
$$

hold true. We recall that, as shown in the previous section, for the error of the best approximation,

$$
E_{m}(f)_{w, 1} \leq \mathcal{C} \frac{\sqrt{a_{m}}}{m}\left\|f^{\prime} \varphi w\right\|_{1}, \quad \mathcal{C} \neq \mathcal{C}(m, f), \quad f \in W_{1}^{1}(w) .
$$

On the other hand, inequality (6.4) holds, mutatis mutandis, for Gaussian rules on bounded intervals related to Jacobi weights. But, as for many exponential weights (see, e.g., [4, 5, 10, $11])$, inequality (6.4) is false in the sense of the following theorem.

THEOREM 6.3 (cf. [14, p. 1661]). Let $w(x)=x^{\gamma} \mathrm{e}^{-x^{-\alpha}-x^{\beta}}, \alpha>0, \beta>1$, and $\gamma \geq 0$. Then, for any $f \in W_{1}^{1}(w)$, we have

$$
\left|e_{m}(f)\right| \leq \mathcal{C} m^{1 / 3} \frac{\sqrt{a_{m}}}{m}\left\|f^{\prime} \varphi w\right\|_{1},
$$


where $\mathcal{C}$ is independent of $m$ and $f$. Moreover, for a sufficiently large $m$ (say $m \geq m_{0}$ ), there exists a function $f_{m}$, with $0<\left\|f_{m}^{\prime} \varphi w\right\|_{1}<+\infty$, and a constant $\mathcal{C} \neq \mathcal{C}\left(m, f_{m}\right)$ such that

$$
\left|e_{m}\left(f_{m}\right)\right| \geq \mathcal{C} m^{1 / 3} \frac{\sqrt{a_{m}}}{m}\left\|f_{m}^{\prime} \varphi w\right\|_{1}
$$

Nevertheless, estimates of the form (6.4) are required in different contexts. To obtain this kind of error estimates, using also an idea from [10], we are going to modify the Gaussian rule.

With $\theta \in(0,1)$ fixed, we define two indexes $j_{1}=j_{1}(m)$ and $j_{2}=j_{2}(m)$ as in (2.3). Then, for a sufficiently large $N$, let $\mathbb{P}_{N}^{*}$ denote the following subset of all polynomials of degree at most $N$,

$$
\mathbb{P}_{N}^{*}=\left\{P \in \mathbb{P}_{N}: P\left(x_{i}\right)=0, x_{i}<x_{j_{1}} \text { or } x_{i}>x_{j_{2}}\right\} .
$$

Naturally, $p_{m}(w) \in \mathbb{P}_{N}^{*}$, for $N \geq m$, and $\theta \in(0,1)$ arbitrary. Now, in analogy with (6.1), we define the new Gaussian rule by means of the equality

$$
\int_{0}^{+\infty} Q_{2 m-1}(x) w(x) \mathrm{d} x=\sum_{k=1}^{m} \lambda_{k}(w) Q_{2 m-1}\left(x_{k}\right)=\sum_{k=j_{1}}^{j_{2}} \lambda_{k}(w) Q_{2 m-1}\left(x_{k}\right),
$$

which holds for every $Q_{2 m-1} \in \mathbb{P}_{2 m-1}^{*}$.

Then, for any continuous function $f$, the "truncated" Gaussian rule is defined as

$$
\int_{0}^{+\infty} f(x) w(x) \mathrm{d} x=\sum_{k=j_{1}}^{j_{2}} \lambda_{k}(w) f\left(x_{k}\right)+e_{m}^{*}(f)
$$

whose error $e_{m}^{*}(f)$ is the difference between the integral and the quadrature sum.

Compared to the Gaussian rule (6.1), in the formula (6.5), the terms of the quadrature sum corresponding to the zeros that are "close" to the MRS numbers are dropped. From the numerical point of view, this fact has two consequences. First, it avoids overflow phenomena (taking into account that, in general, the function $f$ is exponentially increasing at the endpoints of $\mathbb{R}_{+}$). Moreover, it produces a computational saving, which is evident in the numerical treatment of linear functional equations (see [15]).

We are now going to study the behaviour $e_{m}^{*}(f)$ in $C_{\sigma}$ and $W_{r}^{1}(w)$. We will see that the errors $e_{m}(f)$ and $e_{m}^{*}(f)$ have essentially the same behaviour in $C_{\sigma}$ but not in $W_{r}^{1}(w)$ since $e_{m}^{*}(f)$ satisfies (6.4), while $e_{m}(f)$ does not. The behaviour of $e_{m}^{*}(f)$ in $C_{\sigma}$ is given by the following proposition.

Proposition 6.4 (cf. [14, p. 1662]). Assume that $w / \sigma \in L^{1}$. Then, for any $f \in C_{\sigma}$, we get

$$
\left|e_{m}^{*}(f)\right| \leq \mathcal{C}\left\{E_{M}(f)_{\sigma, \infty}+\mathrm{e}^{-c m^{\nu}}\|f \sigma\|_{\infty}\right\}
$$

where $M=\left\lfloor\left(\frac{\theta}{\theta+1}\right) m\right\rfloor, \theta \in(0,1), \nu$ is given by (3.2), $\mathcal{C} \neq \mathcal{C}(m, f)$, and $c \neq c(m, f)$.

In particular, if $f \in W_{r}^{\infty}(\sigma)$, then the inequality (6.6) becomes

$$
\left|e_{m}^{*}(f)\right| \leq \mathcal{C}\left(\frac{\sqrt{a_{m}}}{m}\right)^{r}\|f\|_{W_{r}^{\infty}(\sigma)} .
$$


For smoother functions, the analogue of Theorem 6.2 is given by the following statement.

THEOREM 6.5 (cf. [14, p. 1662]). If the weight $\sigma$ and the function $f$ satisfy the assumption of Theorem 6.2, then, for any $0<\mu<\alpha(1-1 /(2 \beta)) /(\alpha+1 / 2)$ fixed, we get

$$
\left|e_{m}^{*}(f)\right| \leq \mathcal{C}\left[\|f \sigma\|_{\infty}+K(f)\right] \bar{\Gamma}_{m}
$$

where $\lim _{m} \bar{\Gamma}_{m}^{1 / m^{\mu}}=0$ and $\mathcal{C} \neq \mathcal{C}(m, f)$.

For functions $f \in W_{1}^{1}(w)$ or $f \in Z_{s}^{1}(w), 1<s \in \mathbb{R}_{+}$, the following theorem states the required estimates.

THEOREM 6.6 (cf. [14, pp. 1662-1663]). For any $f \in W_{1}^{1}(w)$, we have

$$
\left|e_{m}^{*}(f)\right| \leq \mathcal{C} \frac{\sqrt{a_{m}}}{m}\left\|f^{\prime} \varphi w\right\|_{1}+\mathcal{C} \mathrm{e}^{-c m^{\nu}}\|f w\|_{1} .
$$

Moreover, for any $f \in Z_{s}^{1}(w)$, with $s>1$, we get

$$
\left|e_{m}^{*}(f)\right| \leq \mathcal{C} \frac{\sqrt{a_{m}}}{m} \int_{0}^{\sqrt{a_{m}} / m} \frac{\Omega_{\varphi}^{r}(f, t)_{w, 1}}{t^{2}} \mathrm{~d} t+\mathcal{C} \mathrm{e}^{-c m^{\nu}}\|f w\|_{1},
$$

where $r>s>1$. In both cases $\mathcal{C}$ and $c$ do not depend on $m$ and $f$, and $\nu$ is given by (3.2).

In conclusion, inequality (6.7) is the required estimate and, by (6.8), it can be generalized as

$$
\left|e_{m}^{*}(f)\right| \leq \mathcal{C}\left(\frac{\sqrt{a_{m}}}{m}\right)^{s}\|f\|_{Z_{s}^{1}(w)}, \quad \mathcal{C} \neq \mathcal{C}(m, f),
$$

for $f \in Z_{s}^{1}(w), s>1$. In particular, if $s$ is an integer, recalling (6.7), the Zygmund norm can be replaced by the Sobolev norm.

Finally, we emphasize that the previous estimate cannot be improved since, in these function spaces, $e_{m}^{*}(f)$ converges to 0 with the order of the best polynomial approximation.

6.2. Lagrange interpolation in $L_{\sqrt{w}}^{2}$. Here we want to apply the results in Section 6.1 to estimate the error of the Lagrange interpolation process based on the zeros of $p_{m}(w)$. If $f \in C^{0}\left(\mathbb{R}_{+}\right)$, then the Lagrange polynomial interpolating $f$ at the zeros of $p_{m}(w)$ is defined by

$$
L_{m}(w, f, x)=\sum_{k=1}^{m} l_{k}(w, x) f\left(x_{k}\right), \quad l_{k}(w, x)=\frac{p_{m}(w, x)}{p_{m}^{\prime}\left(w, x_{k}\right)\left(x-x_{k}\right)},
$$

and we are going to study the error $\left\|\left[f-L_{m}(w, f)\right] \sqrt{w}\right\|_{2}$ for different function classes. Since

$$
\left\|L_{m}(w, f) \sqrt{w}\right\|_{2}^{2}=\sum_{k=1}^{m} \frac{\lambda_{k}(w)}{w\left(x_{k}\right)}(f \sqrt{w})^{2}\left(x_{k}\right)
$$

and we are dealing with an unbounded interval, we cannot expect an analogue of the theorem by Erdős and Turán [6]. On the other hand, if $f \in C_{\tilde{u}}$, with $\tilde{u}(x)=(1+x)^{\delta} \sqrt{w(x)}, \delta>1 / 2$, then it is easily seen that

$$
\left\|\left[f-L_{m}(w, f)\right] \sqrt{w}\right\|_{2} \leq \mathcal{C} E_{m-1}(f)_{\tilde{u}, \infty}, \quad \mathcal{C} \neq \mathcal{C}(m, f) .
$$

Nevertheless, as for the Gaussian formula, if $f \in W_{1}^{2}(\sqrt{w})$, then $L_{m}(w, f)$ has not an optimal behaviour, i.e., an estimate of the form

$$
\left\|\left[f-L_{m}(w, f)\right] \sqrt{w}\right\|_{2} \leq \mathcal{C} \frac{\sqrt{a_{m}}}{m}\left\|f^{\prime} \varphi \sqrt{w}\right\|_{2}, \quad \mathcal{C} \neq \mathcal{C}(m, f),
$$


does not hold. In order to overcome this gap, for any $f \in C^{0}\left(\mathbb{R}_{+}\right)$, we introduce the following "truncated" Lagrange polynomial

$$
L_{m}^{*}(w, f, x)=\sum_{k=j_{1}}^{j_{2}} l_{k}(w, x) f\left(x_{k}\right),
$$

where $j_{1}$ and $j_{2}$ are given by (2.3).

Naturally, in general $L_{m}^{*}(w, P) \neq P$ for arbitrary polynomials $P \in \mathbb{P}_{m-1}$ (for example, $\left.L_{m}(w, \mathbf{1}) \neq \mathbf{1}\right)$. But $L_{m}^{*}(w, Q)=Q$ for any $Q \in \mathbb{P}_{m-1}^{*}$ and $L_{m}^{*}(w, f) \in \mathbb{P}_{m-1}^{*}$ for any $f \in C^{0}\left(\mathbb{R}_{+}\right)$. Therefore, the operator $L_{m}^{*}(w)$ is a projector from $C^{0}\left(\mathbb{R}_{+}\right)$into $\mathbb{P}_{m-1}^{*}$.

Moreover, considering the weight

$$
\tilde{u}(x)=(1+x)^{\delta} \sqrt{w(x)}, \quad \delta>0,
$$

we can show that every function $f \in L_{\tilde{u}}^{p}$ can be approximated by polynomials of $\mathbb{P}_{m}^{*}$. To this aim we define

$$
\widetilde{E}_{m}(f)_{\tilde{u}, p}=\inf _{P \in \mathbb{P}_{m}^{*}}\|(f-P) \tilde{u}\|_{p}, \quad 1 \leq p \leq+\infty .
$$

LEMMA 6.7 (cf. [14, p. 1664]). For any $f \in L_{\tilde{u}}^{p}$, where $\tilde{u}$ is given by (6.10) and $1 \leq p \leq+\infty$, we have

$$
\widetilde{E}_{m}(f)_{\tilde{u}, p} \leq \mathcal{C}\left\{E_{M}(f)_{\tilde{u}, p}+\mathrm{e}^{-c m^{\nu}}\|f \tilde{u}\|_{p}\right\},
$$

where $M=\left\lfloor\left(\frac{\theta}{\theta+1}\right) m\right\rfloor, \theta \in(0,1), \nu$ is given by (3.2), $\mathcal{C} \neq \mathcal{C}(m, f)$, and $c \neq c(m, f)$.

As an immediate consequence of the previous lemma and equality (6.9), we get the following:

Proposition 6.8 (cf. [14, p. 1664]). For any $f \in C_{\tilde{u}}$, with $\tilde{u}$ as (6.10), $\delta>1 / 2$, we have

$$
\left\|\left[f-L_{m}^{*}(w, f)\right] \sqrt{w}\right\|_{2} \leq \mathcal{C}\left\{E_{M}(f)_{\tilde{u}, \infty}+\mathrm{e}^{-c m^{\nu}}\|f \tilde{u}\|_{\infty}\right\},
$$

where $M=\left\lfloor\left(\frac{\theta}{\theta+1}\right) m\right\rfloor, \theta \in(0,1), \nu$ is given by (3.2), $\mathcal{C} \neq \mathcal{C}(m, f)$, and $c \neq c(m, f)$.

We are going to study the behaviour of the sequence $\left\{L_{m}^{*}(w)\right\}_{m}$ in the Sobolev spaces $W_{r}^{2}(\sqrt{w})$, which is interesting in different contexts. We observe that, since no results concerning the sequence of the Fourier sum $\left\{S_{m}(w)\right\}_{m}$ are known, we cannot deduce the behaviour of $\left\{L_{m}^{*}(w)\right\}_{m}$ from that of $\left\{S_{m}(w)\right\}_{m}$. Therefore, we need a different approach. The following theorem describes the behaviour of the operator $L_{m}^{*}(w)$ in different function spaces.

THEOREM 6.9 (cf. [14, p. 1664]). Assume $f \in L_{\sqrt{w}}^{2}$ and

$$
\int_{0}^{1} \frac{\Omega_{\varphi}^{r}(f, t)_{\sqrt{w}, 2}}{t^{3 / 2}} \mathrm{~d} t<+\infty, \quad r \geq 1 .
$$

Then we have

$$
\left\|\left[f-L_{m}^{*}(w, f)\right] \sqrt{w}\right\|_{2} \leq \mathcal{C}\left\{\left(\frac{\sqrt{a_{m}}}{m}\right)^{\frac{1}{2}} \int_{0}^{\frac{\sqrt{a_{m}}}{m}} \frac{\Omega_{\varphi}^{r}(f, t)_{\sqrt{w}, 2}}{t^{3 / 2}} \mathrm{~d} t+\mathrm{e}^{-c m^{\nu}}\|f \sqrt{w}\|_{2}\right\},
$$

where $\nu$ is given by (3.2) and the constants $\mathcal{C}, c$ are independent of $m$ and $f$. 


\section{ETNA}

Kent State University and

Johann Radon Institute (RICAM)

Note that, by Corollary 5.4, assumption (6.11) implies $f \in C^{0}\left(\mathbb{R}_{+}\right)$. The error estimate (6.12) has interesting consequences. Firstly, if

$$
\sup _{t>0} \frac{\Omega_{\varphi}^{r}(f, t)_{\sqrt{w}, 2}}{t^{s}} \mathrm{~d} t<+\infty, \quad r>s>1 / 2,
$$

i.e., $f \in Z_{s}^{2}(\sqrt{w})$, then the order of convergence of the process is $\mathcal{O}\left(\left(\sqrt{a_{m}} / m\right)^{s}\right)$. If, instead, $f \in W_{r}^{2}(\sqrt{w})$, with $r \geq 1$ an integer, then we have

$$
\left\|\left[f-L_{m}^{*}(w, f)\right] \sqrt{w}\right\|_{2} \leq \mathcal{C}\left(\frac{\sqrt{a_{m}}}{m}\right)^{r}\|f\|_{W_{r}^{2}(\sqrt{w})} .
$$

This means that the process converges with the error of the best approximation for the considered classes of functions.

Secondly, we are now able to show the uniform boundedness of the sequence $\left\{L_{m}^{*}(w)\right\}$ in the Sobolev spaces.

THEOREM 6.10 (cf. [14, p. 1665]). With the previous notation, for any $f \in W_{r}^{2}(\sqrt{w})$, $r \geq 1$, we have

$$
\sup _{m}\left\|L_{m}^{*}(w, f)\right\|_{W_{r}^{2}(\sqrt{w})} \leq \mathcal{C}\|f\|_{W_{r}^{2}(\sqrt{w})}, \quad \mathcal{C} \neq \mathcal{C}(f) .
$$

Moreover, for any $f \in W_{s}^{2}(\sqrt{w}), s>r$, we have

$$
\left\|f-L_{m}^{*}(w, f)\right\|_{W_{r}^{2}(\sqrt{w})} \leq \mathcal{C}\left(\frac{\sqrt{a_{m}}}{m}\right)^{s-r}\|f\|_{W_{s}^{2}(\sqrt{w})}, \quad \mathcal{C} \neq \mathcal{C}(m, f) .
$$

REMARK 6.11. In all the estimates for $e_{m}^{*}(f)$ and $\left(f-L_{m}^{*}(w, f)\right)$, a constant $\mathcal{C} \neq \mathcal{C}(m, f)$ appears. We have not indicated the dependence on the parameter $\theta \in(0,1)$ since $\theta$ is fixed. Nevertheless, it is useful to observe that $\mathcal{C}=\mathcal{C}(\theta)=\mathcal{O}\left((\theta /(1-\theta))^{2}\right)$. So, it is clear that the parameter $\theta$ cannot assume the value 0 or 1 , and the "truncation" is necessary in this sense (see [14] for more details).

\section{REFERENCES}

[1] R. AlJARRAH, Error estimates for Gauss-Jacobi quadrature formulae with weights having the whole real line as their support, J. Approx. Theory, 30 (1980), pp. 309-314.

[2] - An error estimate for Gauss-Jacobi quadrature formula with the Hermite weight $w(x)=\exp \left(-x^{2}\right)$, Publ. Inst. Math. (Beograd) (N.S.), 33 (1983), pp. 17-22.

[3] A. S. Cvetković and G. V. Milovanović, The Mathematica Package "OrthogonalPolynomials", Facta Univ. Ser. Math. Inform., 9 (2004), pp. 17-36.

[4] M. C. De Bonis, G. Mastroianni, AND I. Notarangelo, Gaussian quadrature rules with exponential weights on $(-1,1)$, Numer. Math., 120 (2012), pp. 433-464.

[5] B. Della Vecchia and G. Mastroianni, Gaussian rules on unbounded intervals, J. Complexity, 19 (2003), pp. 247-258.

[6] P. ERDôS AND P. TURÁN, On interpolation. I. Quadrature- and mean-convergence in the Lagrangeinterpolation, Ann. Math., 38 (1937), pp. 142-155.

[7] A. L. LeVin And D. S. Lubinsky, Orthogonal Polynomials for Exponential Weights, Springer, New York, 2001.

[8] D.S. LUBINSKY, Geometric convergence of Lagrangian interpolation and numerical integration rules over unbounded contours and intervals, J. Approx. Theory, 39 (1983), pp. 338-360.

[9] G. Mastroianni And G.V. Milovanović, Interpolation Processes. Basic Theory and Applications, Springer, Berlin, 2008.

[10] G. Mastroianni and G. Monegato, Truncated quadrature rules over $(0, \infty)$ and Nyström type methods, SIAM J. Numer. Anal., 41 (2003), pp. 1870-1892. 
ETNA

Kent State University and

Johann Radon Institute (RICAM)

POLYNOMIAL APPROXIMATION WITH POLLACZECK-LAGUERRE WEIGHTS

[11] G. Mastroianni and I. Notarangelo, A Lagrange-type projector on the real line, Math. Comp., 79 (2010), pp. 327-352.

[12] - Embedding theorems with an exponential weight on the real semiaxis, Electron. Notes Disc. Math., 43 (2013), pp. 15-160.

[13] - Polynomial approximation with an exponential weight on the real semiaxis, Acta Math. Hungar., 142 (2014), pp. 167-198.

[14] G. Mastroianni, I. Notarangelo, And G.V. Milovanović, Gaussian quadrature rules with an exponential weight on the real semiaxis, IMA J. Numer. Anal., 34 (2014), pp. 1654-1685.

[15] _ A Nyström method for a class of Fredholm integral equations on the real semiaxis, Calcolo, 54 (2017), pp. 567-585.

[16] G. Mastroianni, I. Notarangelo and J. Szabados, Polynomial inequalities with an exponential weight on $(0,+\infty)$, Mediterr. J. Math., 10 (2013), pp. 807-821.

[17] G. Mastroianni And J. Szabados, Polynomial approximation on the real semiaxis with generalized Laguerre weights, Stud. Univ. Babeş-Bolyai Math., 52 (2007), pp. 105-128.

[18] G. V. Milovanović, Construction and applications of Gaussian quadratures with nonclassical and exotic weight function, Stud. Univ. Babeş-Bolyai Math., 60, (2015), pp. 211-233.

[19] G. V. Milovanović AND A. S. CVETKOVIĆ, Special classes of orthogonal polynomials and corresponding quadratures of Gaussian type, Math. Balkanica, 26 (2013), pp. 169-184.

[20] J.V. USPENSKY, On the convergence of quadrature formulas related to an infinite interval, Trans. Amer. Math. Soc., 30 (1928), pp. 542-559. 